\title{
Will Genotyping Replace Serology in Future Routine Blood Grouping? - Opinion 1
}

\author{
Franz F. Wagner \\ DRK-Blutspendedienst NSTOB, Zentralinstitut Springe, Germany
}

Currently, genotyping is complementing serology. There are special situations in which molecular typing is undoubtedly superior to serology $[1,2]$, and the description of an D or ABO variant antigen would be considered incomplete by most experts if a molecular characterization is lacking. Molecular typing has successfully been used as complement to serologic donor typing of specific donor groups and gained routine status in some blood services. Typical indications include $R H D$ PCR of D-negative donors to exclude DEL, screening of donors for rare blood group constellations, and typing donors for antigens apart from $\mathrm{ABO}, \mathrm{RH}$ and $\mathrm{K}$.

I expect that these focused molecular typing applications will increasingly be used and partly replace serology: For example, in blood donors, a combination of antigen $\mathrm{D}$ detection by direct agglutination with $R H D$ PCR is likely to be superior to $\mathrm{D}$ testing in the indirect antiglobulin test. The increasing availability of molecularly typed units will allow molecular matching of donors and patients in situations in which the current standard does not include any matching. Possible applications might be matching of chronically transfused or RHimmunized patients for antigens $\mathrm{Fy}^{\mathrm{a} / \mathrm{b}}$ and $\mathrm{Jk}^{\mathrm{a} / \mathrm{b}}$ in the absence of evidence for immunization against these antigens or $\mathrm{Jk}^{\mathrm{a}}$ negative transfusion of $\mathrm{Jk}^{\mathrm{a}}$-negative, previously transfused patients. Any discussion on a residual error rate of molecular typing is absurd in these situations because molecular matching is obviously better than no matching. Already now, the error rate of some molecular typing methods is lower than that of serologic testing [3]. I would not be surprised if, within a few years, it will become accepted that molecular matching can fully or partially replace serologic matching even in preimmunized patients.

The outlined applications focus on a limited number of patients, and they may be accomplished without typing all donors. While some of these applications clearly improve the quality of transfusion support without possible adverse effects, cost efficiency will remain a matter. Focusing typing to specific donor groups like frequent donors, blood group $\mathrm{O}$ and A donors, or, considering platelet antigens, apheresis donors may enhance cost efficiency. Likewise, allele frequencies vastly differ between populations, and a population-adapted typing strategy likely outperforms an 'universal' typing strategy designed for all populations concerning cost efficiency. Only in the far future, a single 'fail-safe' typing run including whole gene sequencing may be expected to be used for all populations.

Will we molecularly type all donors in the far future, fully replacing serology at least for some donations? Possibly yes, but it will be a very long way. The most likely scenario is that one day, molecular typing gets cheaper than the serologic typing for $\mathrm{ABO}$ and $\mathrm{D}$ currently performed for repeat donors. It will be hard to argue that it is unsafe to release a molecularly typed unit of a repeat donor whose molecular type has been confirmed serologically in the initial donations and whose molecular footprint is identical to the previous one. However, considering the extremely low cost of an ABO/D check, at this day, the molecular techniques will surely differ from current methods.

A less realistic scenario often suggested is an era of molecular transfusion medicine, with all donors and patients molecularly typed for all relevant antigens and units used according to a molecular cross-match. At first glance, serology might be dropped, which would allow enormous savings, and these savings as well as the expected improved quality of transfusion support without immunologically mediated hemolytic transfusion reactions might make molecular typing cost-efficient, even if the typing costs remains higher than current serologic blood group determination. Honestly, I do not believe that this scenario will become reality.

Already now, in Germany all units are typed for antigens $\mathrm{CcEe}$ and $\mathrm{K}$, but many hospitals do not use that information

\section{KARGER}

Fax +497614520714

Information@Karger.de

www.karger.com (c) 2009 S. Karger GmbH, Freiburg

Accessible online at:

www.karger.com/tmh
PD Dr. Franz F. Wagner

DRK-Blutspendedienst NSTOB, Zentralinstitut Springe, Germany

Eldagsener Straße 38, 31832 Springe

franz.wagner@bsd-nstob.de 
for 'random' patients and just match by $\mathrm{ABO}$ and $\mathrm{D}$. There are too many different RH phenotypes. Inevitably, some patients are not transfused with an identical $\mathrm{RH}$ phenotype, but with a compatible $\mathrm{RH}$ phenotype, like CCD.ee for CcDee patients. Thus, the almost 'universal' CCD.ee (and ccD.EE) units are in high demand, while CcD.Ee units accumulate. As alloimunizations even to $\mathrm{RH}$ antigens occur in a minority of patients only, there is no perceived need for matching of all patient groups.

It is unrealistic to believe that clinicians who deliberately do not match for $\mathrm{RH}$ antigens and $\mathrm{K}$ antigens will match for Fy, Jk, and $\mathrm{S}$ antigens. Considering more antigens makes the situation more complicated because getting a full match is increasingly difficult. In contrast, the safety gain of matching for $\mathrm{RH}, \mathrm{K}, \mathrm{Fy}, \mathrm{Jk}$, and $\mathrm{S}$ compared to matching for $\mathrm{RH}$ and $\mathrm{K}$ is likely less than that of matching for $\mathrm{RH}$ and $\mathrm{K}$ compared to matching for $\mathrm{ABO}$ and $\mathrm{D}$ only. An $\mathrm{A} \mathrm{CcDEe} \mathrm{Jk}(\mathrm{a}+\mathrm{b}+)$ $\mathrm{Fy}(\mathrm{a}+\mathrm{b}+)$ Ss unit will be compatible for $1.2 \%$ of patients only, and for some patients, like those of phenotype O ccD.EE $\mathrm{Fy}(\mathrm{a}+\mathrm{b}-) \mathrm{Jk}(\mathrm{a}+\mathrm{b}-)$ ss, compatible units occur with a frequency of only about 1:20,000. It will be impossible to store such units in a peripheral hospital in sufficient number. There are bleeding patients that cannot wait until matched units arrive. In addition, it is unlikely that the clinicians will know the exact number of needed units in advance, and they will order more units of a strange phenotype than really needed, leading to the accumulation of such units: Currently almost twice as much units are cross-matched than transfused. Before throwing away units with less suitable antigen pattern, these units will be transfused without a full molecular match. As a consequence, the hospitals will have to keep serology to detect patients with irregular antibodies who really need matched blood. However, as long the hospitals have to keep serology, they will find no convincing argument to switch to full matching for most patient groups and will continue with antibody screen and partial matching. Other arguments for keeping serology are those already now supporting the use of a serologic cross-match: antibodies to rare antigens and the possibility of a sample mix-up.

A third scenario for the introduction of general molecular typing would include molecular typing as an 'add-on' to testing for 'soft' genetic diseases like hemochromatosis used as donor incentive. This scenario might be a double-edged sword: The extensive counseling needed for predictive genetic testing (as well as long-term DNA storage) will be difficult to be accomplished in current blood donation settings outside university hospitals. Furthermore, while some donors might be interested in getting tested, others might be worried by the possibility of predictive genetic testing and stay away from the donation site.

So in conclusion, already now molecular typing is replacing serology in specific situations. Its use will increase and improve the quality of transfusion support. However, I believe that molecular testing will not be used for all donors until it gets cheaper or at least comparably cheap as serology of the typical transfusion-relevant antigens.

\section{References}

1 Flegel WA, Wagner FF: Genotyping of red blood cell, granulocyte and platelet antigens: current applications in the German-speaking countries; in Scharf RD (ed): Progress and Challenges in Transfusion Medicine, Hemostatis and Hemotherapy. Freiburg i.Br., Karger, 2009, pp 189-198.
2 Legler TJ, Kroll H, Wagner FF, Flegel WA, Hallensleben M: Indikation und Durchführung einer Genotypisierung erythrozytärer Antigene. Infus Ther Transfus Med 2000;27:215-216.
3 Avent ND on behalf of the Bloodgen consortium. The Bloodgen project of the European Union; in Scharf RD (ed): Progress and Challenges Transfusion Medicine, Hemostatis and Hemotherapy. Freiburg i.Br., Karger, 2009, pp 178-188. 